\title{
Micromechanical modelling of damage induced by delayedettringite formation in concrete
}

\author{
Alexandre Yammine ${ }^{l}$, François Bignonnet ${ }^{l, 1}$, Nordine Leklou $^{l}$, and Marta Choinska $^{l}$ \\ ${ }^{1}$ GeM, UMR CNRS 6183, Université de Nantes, Ecole Centrale Nantes, 44600 Saint-Nazaire, France
}

\begin{abstract}
A multi-scale poromechanical model of damage induced by Delayed Ettringite Formation (DEF) as a consequence of progression of micro-cracks at the fine aggregate scale is developed. The aim is to link the DEF-induced expansion at both the microscopic and macroscopic scales to the loss of stiffness of the mortar and the increase of its diffusion coefficient. At the microscopic scale, mortar is assumed to be constituted of three phases: cement paste, sand and micro-cracks. Damage is assumed to be driven by a free expansion of cement paste due to ettringite crystallization pressures in small capillary pores, at a lower scale. The corresponding homogenised poroelastic properties are estimated along with the diffusion coefficient by resorting either to a Mori-Tanaka scheme or to a self-consistent scheme, as a function of paste and aggregate properties as well as on the density of micro-cracks. The latter is assumed to be an evolving internal variable in order to model DEF-induced damage in the mortar. As the DEF-induced expansive free strain in the cement paste is restrained by the sand particles, internal stresses arise in the mortar. The corresponding free energy can be partially released by an increase in the micro-cracks density by analogy with the energy restitution rate of linear elastic fracture mechanics. The role of the damage criterion adopted on the thermodynamic force associated with micro-cracks density increase is investigated.
\end{abstract}

\section{Introduction}

Delayed ettringite formation (DEF) is a concrete pathology that may occur in concrete structures subjected to temperature above $65-70^{\circ} \mathrm{C}$ at early age, then left in contact with water. It manifests by late crystallization of ettringite in the pore space, leading to concrete expansion, micro-craking, loss of mechanical performances and increase in transport properties $[1,2]$.

DEF involves coupled phenomena at several length scales such as chemically-driven crystallization pressures, ionic transport by diffusion, poro-mechanics and damage. According to [3, 4], DEF crystallization pressures arise first in small capillary pores, where sulfates are available from monosulfoaluminate or adsorbed in C-S-H. In those pores, the hydroxyl activity drop due to the macroscopic alkali leaching leads to an increase of the solubility product of ettringite over its equilibrium value. Ettringite crystallizes provided

\footnotetext{
${ }^{1}$ Corresponding author: francois.bignonnet@univ-nantes.fr
} 
the supersaturation is high enough to overcome surface tensions, leading to crystallization pressures inversely proportional to pore throat size [4].

Several theories are then possible. According to [3], these pressures may lead to a uniform expansion of cement paste, which is restrained by aggregates, and produces internal stresses. The latter are eventually released by diffuse microcracking responsible for the apparent damage. According to [1], large crystals of ettringite instead grow preferentially in weaknesses such as macropores, cracks and interface between paste and aggregate. [5] suggested a combined mechanism in which uniform expansion of cement paste first initiates microcracking, which is progressively filled and increased by new products. In all cases, a feedback loop is then initiated as microcracking leads to a rise in diffusion coefficient [6], which accelerates alkali leaching and thus ettringite crystallization, until reactants are consumed.

The coupled modeling of these phenomena, as addressed by e.g. [7-9], is beyond the scope of the present contribution. We instead focus on one elementary brick of these coupled models: damage induced by a possible free expansion of cement paste restrained by aggregates, and its consequences on both mechanical and transport properties. Multiscale modeling of damage due to expansive reactions has been investigated by e.g. [10-14] using continuum micromechanics and $[15,16]$ by numerical simulations. We turn to continuum micromechanics which allows an analytical treatment of damage due to microcracking [17-22] and of coupling between mechanical damage and transport properties [18, 23-27].

Sect. 2 presents a micromechanical model of mortar subjected to a free expansion of cement paste and the homogenization of both poro-elastic and diffusive properties, as a function of micro-crack density, for two types of homogenization schemes. The development of damage by progression of microcracking is exposed in Sect. 3. The role of the different damage evolution laws suggested by previous investigators is illustrated and compared to experimental results on Young's modulus drop as a function of the macroscopic expansion.

\section{Homogenization of poro-mechanical and transport properties}

Notations: The second order identity tensor is denoted $\mathbf{1}$ and any isotropic second order tensor decomposes as $\boldsymbol{D}=D \mathbf{1}$. The fourth order identity tensor is denoted $\mathbf{I}$ and any isotropic fourth order tensor decomposes as $\boldsymbol{C}=C_{\mathrm{sph}} \mathbf{J}+C_{\mathrm{dev}} \mathbf{K}$ where $\mathbf{J}=1 / 3 \quad \mathbf{1} \otimes \mathbf{1}$ is the spherical projector and $\mathbf{K}=\mathbf{I}-\mathbf{J}$ is the deviatoric projector. The isotropic fourth order tensors $\mathbf{J}$ and $\mathbf{K}$ are orthogonal and idempotent for the double dot " : " product so that $\boldsymbol{A}: \boldsymbol{B}$ $=A_{\mathrm{sph}} B_{\mathrm{sph}} \mathbf{J}+A_{\mathrm{dev}} B_{\mathrm{dev}} \mathbf{K}$ for any isotropic fourth order tensors $\boldsymbol{A}, \boldsymbol{B}$ and the inverse of $\boldsymbol{A}$ is $\boldsymbol{A}^{-1}$ $=\left(A_{\mathrm{sph}}\right)^{-1} \mathbf{J}+\left(A_{\mathrm{dev}}\right)^{-1} \mathbf{K}$.

\subsection{Multiscale description of the mortar}

The mortar is described at two separate length scales. At the microscopic scale, a representative volume element (RVE) $\Omega$ of the mortar comprises cement paste (index $i=$ $p$ ), sand aggregates (index $i=s$ ) and - as damage develops - microcracks (index $i=c$ ), see figure 1 . The volume fraction of each constituent $i \quad\{p, s, c\}$ is denoted $f_{\mathrm{i}}$ and the domain they occupy within $\Omega$ is $\Omega_{\mathrm{i}}$. For the diffusion, Fick's law is assumed with an isotropic diffusion coefficient $D_{\mathrm{i}}=D_{\mathrm{i}} \mathbf{1}$ in each phase $i$ relating the molar flux $\boldsymbol{j}$ to the concentration gradient $\nabla \boldsymbol{c}$ of a chemical specie. The mechanical constitutive law of each phase $i$ is assumed isotropic linear elastic characterized by a stiffness tensor $\boldsymbol{C}_{\mathrm{i}}=3 k_{\mathrm{i}} \mathbf{J}+2 \mu_{\mathrm{i}} \mathbf{K}$ where $k_{\mathrm{i}}$ and $\mu_{\mathrm{i}}$ are the bulk and shear moduli, relating the Cauchy stress tensor $\boldsymbol{\sigma}$ to the linearized 
strain tensor $\boldsymbol{\varepsilon}$. In addition, the cement paste phase is assumed to undergo an isotropic free expansive strain $\boldsymbol{\varepsilon}_{\mathrm{f}}$ and the crack stiffness is $\boldsymbol{C}_{\mathrm{c}}=0$ for opened cracks.

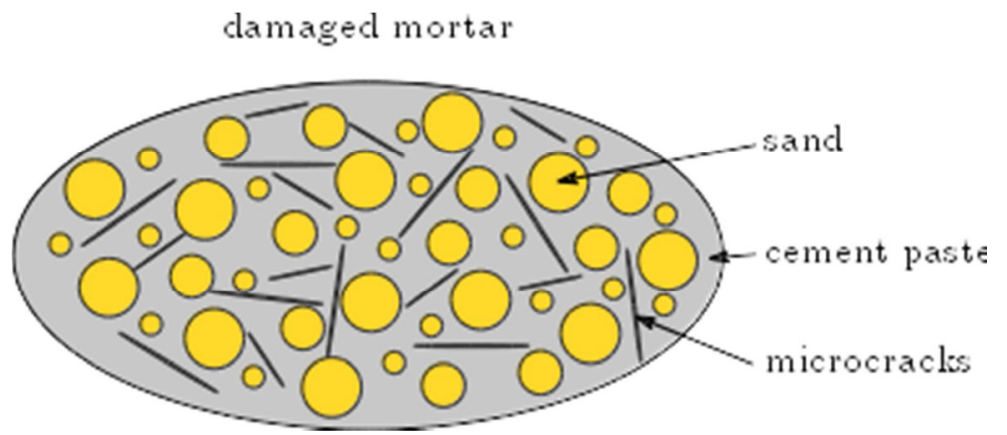

Fig. 1. Schematic view of a representative volume element of damaged mortar

At the macroscopic scale, the mortar may undergo some macroscopic stress $\Sigma$ and strain $\boldsymbol{E}$ as well as some macroscopic molar flux $\boldsymbol{J}$ and concentration gradient $\boldsymbol{\nabla} \boldsymbol{C}$. The macroscopic constitutive laws relating these quantities may be derived from the above microscopic properties by homogenization. To do so, in the framework of continuum micromechanics [28] with uniform strain (resp. concentration gradient) boundary conditions, a displacement $\boldsymbol{u}=\boldsymbol{E} \cdot \boldsymbol{x}$ (resp. a concentration $c=\boldsymbol{\nabla} \boldsymbol{C} \cdot \boldsymbol{x}$ ) is imposed at the boundary $+\Omega$ of the RVE, where $\boldsymbol{x}$ is the position vector at the microscopic scale. The macroscopic strain $\boldsymbol{E}$ (resp. concentration gradient $\nabla \boldsymbol{C}$ ) is then related to its microscopic counterpart by the volume averaging rule (1a). By virtue of the conservation law $\nabla \cdot \sigma=0$ (resp. $\nabla \cdot j=0$ ), the energy equivalence of the microscopic and macroscopic point of views implies as well that the macroscopic stress $\Sigma$ (resp. molar flux $\boldsymbol{J}$ ) corresponds to the volume average of its microscopic counterpart (1b)

$$
\begin{gathered}
\boldsymbol{E}=\overline{\boldsymbol{\epsilon}} ; \quad \boldsymbol{\nabla C}=\overline{\boldsymbol{\nabla} \boldsymbol{c}} \\
\Sigma=\overline{\boldsymbol{\sigma}} ; \quad \boldsymbol{J}=\overline{\boldsymbol{j}} \\
\text { where } \bar{\bullet}=\frac{1}{|\Omega|} \int_{\Omega} \bullet(\boldsymbol{x}) \mathrm{d} V=\sum_{i \in\{p, s, c\}} f_{i} \bar{\bullet}^{i} \text { and } \bar{\bullet}^{i}=\frac{1}{\left|\Omega_{i}\right|} \int_{\Omega_{i}}^{\bullet}(\boldsymbol{x}) \mathrm{d} V
\end{gathered}
$$

The homogenized constitutive laws may then formally be obtained by resolution of the mechanical and diffusive homogenization problems defined by:

$$
\left\{\begin{array}{lr}
\boldsymbol{\nabla} \cdot \boldsymbol{\sigma}=0 & (\Omega) \\
\boldsymbol{\sigma}=\mathbb{C}_{i}: \boldsymbol{\epsilon} \quad \forall i \in\{s, c\} & \left(\Omega_{i}\right) \\
\boldsymbol{\sigma}=\mathbb{C}_{p}:\left(\boldsymbol{\epsilon}-\boldsymbol{\epsilon}_{f}\right)=\mathbb{C}_{p}: \boldsymbol{\epsilon}-p \mathbf{1} & \left(\Omega_{p}\right) \\
\boldsymbol{\epsilon}=\operatorname{sym}(\boldsymbol{\nabla} \boldsymbol{u}) & (\Omega) \\
\boldsymbol{u}=\boldsymbol{E} \cdot \boldsymbol{x} & (\partial \Omega)
\end{array} \quad ; \quad\left\{\begin{array}{l}
\boldsymbol{\nabla} \cdot \boldsymbol{j}=0 \\
\boldsymbol{j}=-\boldsymbol{D}_{i} \cdot \boldsymbol{\nabla} c \quad \forall i \in\{p, s, c\} \\
c=\boldsymbol{\nabla} \boldsymbol{C} \cdot \boldsymbol{x}
\end{array}\right.\right.
$$

To draw an analogy with poroelasticity in what follows, we introduce the notation $p=3 k_{\mathrm{p}} \varepsilon_{\mathrm{f}}$ which corresponds to the pressure generated in the cement paste if its free expansion $\varepsilon_{\mathrm{f}}$ where prevented. By linearity of the homogenization problems (2), there are strain (resp. concentration gradient) concentration tensors linearly relating the local fields to the loading parameters $\boldsymbol{E}$ and $p($ resp. $\boldsymbol{\nabla} \boldsymbol{C})$ : 


$$
\boldsymbol{\epsilon}(\boldsymbol{x})=\mathbb{A}(\boldsymbol{x}): \boldsymbol{E}-\boldsymbol{a}(\boldsymbol{x}) p \quad ; \quad \boldsymbol{\nabla} c(\boldsymbol{x})=\boldsymbol{A}(\boldsymbol{x}) \cdot \boldsymbol{\nabla C}
$$

Gathering the averaging rules (1b) with the local constitutive laws yields the relationships between the loading parameters and the macroscopic stress (resp. molar flux). These correspond to the sought-for macroscopic constitutive laws:

$$
\left\{\begin{aligned}
\boldsymbol{\Sigma} & =\mathbb{C}^{\text {hom }}: \boldsymbol{E}-b^{\mathrm{hom}} p \mathbf{1} \\
\Delta f_{p} & =b^{\text {hom }} \mathbf{1}: \boldsymbol{E}+p / N^{\text {hom }} \quad ; \quad \boldsymbol{J}=-\boldsymbol{D}^{\text {hom }} \cdot \boldsymbol{\nabla C}
\end{aligned}\right.
$$

where:

$$
\begin{array}{ll}
\mathbb{C}^{\text {hom }}=\overline{\mathbb{C}: \mathbb{A}} & \boldsymbol{D}^{\text {hom }}=\overline{\boldsymbol{D} \cdot \boldsymbol{A}} \\
b^{\text {hom }}=\operatorname{sph}\left(f_{p} \overline{\mathbb{A}}^{p}\right)=\frac{1}{3} \mathbf{1}: \overline{\mathbb{C}: \boldsymbol{a}}+f_{p} & 1 / N^{\text {hom }}=-f_{p} \mathbf{1}: \overline{\boldsymbol{a}}^{p}
\end{array}
$$

$\boldsymbol{C}^{\text {hom }}$ and $\boldsymbol{D}^{\text {hom }}$ are the homogenized stiffness and diffusion coefficient; $b^{\text {hom }}, N^{\text {hom }}$ are the analogue to the Biot coefficient and Biot modulus and $\Delta f_{\mathrm{p}}$ is the variation of $f_{\mathrm{p}}$.

\subsection{Classical micromechanical estimates of the homogenized properties}

The homogenized properties (5) depend on the microstructure parameters described in Sect. 2.1 as well as on its precise morphology. Continuum micromechanics provides means to explicitly estimate the homogenized properties from a restricted set of microstructure information, as shortly exposed below.

In Eshelby-based homogenization schemes, the averages of the strain and concentration gradient concentration tensor in each inclusion phase $i$ are estimated from the solutions to auxiliary problems - the so-called Eshelby problems - each featuring a single inclusion of uniform stiffness $\boldsymbol{C}_{\mathrm{i}}$ (resp. diffusivity $\boldsymbol{D}_{\mathrm{i}}$ ) within an infinite matrix of stiffness $\boldsymbol{C}_{0}$ (resp. diffusivity $\boldsymbol{D}_{0}$ ) subjected to a remote load. In the case where the inclusion is ellipsoidal, the strain (resp. concentration gradient) within the inclusion is uniform [29] and depends on the remote strain $\boldsymbol{E}_{\infty}$ (resp. remote concentration gradient $\boldsymbol{\nabla} \boldsymbol{C}_{\infty}$ ) by:

$$
\begin{gathered}
\boldsymbol{\epsilon}=\mathbb{L}_{i}^{0}: \boldsymbol{E}_{\infty} \quad ; \quad \boldsymbol{\nabla} \boldsymbol{c}=\boldsymbol{L}_{i}^{0}: \boldsymbol{\nabla} \boldsymbol{C}_{\infty} \\
\text { where } \quad \mathbb{L}_{i}^{0}=\left[\mathbb{I}+\mathbb{P}_{i}^{0}:\left(\mathbb{C}_{i}-\mathbb{C}_{0}\right)\right]^{-1} \quad ; \quad \boldsymbol{L}_{i}^{0}=\left[\mathbf{1}+\boldsymbol{P}_{i}^{0}:\left(\boldsymbol{D}_{i}-\boldsymbol{D}_{0}\right)\right]^{-1}
\end{gathered}
$$

where $\boldsymbol{P}_{\mathrm{i}}^{0}$ is the Hill tensor which depends the shape (but not the size) of the inclusion $i$ and the stiffness $\boldsymbol{C}_{0}$ of the matrix, and similarly for diffusion. For sand grains modelled as spheres in an isotropic medium of bulk and shear moduli $k_{0}, \mu_{0}$ and diffusion coefficient $D_{0}$, the Hill tensors are:

$$
\mathbb{P}_{s}^{0}=\frac{1}{3 k_{0}+4 \mu_{0}} \mathbb{J}+\frac{3}{5} \frac{k_{0}+2 \mu_{0}}{\mu_{0}\left(3 k_{0}+4 \mu_{0}\right)} \mathbb{K} \quad ; \quad \boldsymbol{P}_{s}^{0}=\frac{1}{3 D_{0}} \mathbf{1}
$$

Cracks are modelled as penny-shaped spheroids of radius $a$ and thickness $b$, with an isotropic distribution of orientations. The aspect ratio $\omega=b / a$ of the oblate spheroids used to model cracks is assumed vanishing: $\omega \varphi 1$. In this limiting case, the tensor $\boldsymbol{L}_{\mathrm{c}}{ }^{0}$ behaves as $\mathrm{O}\left(\omega^{-1}\right)$ while the volume fraction of crack $f_{\mathrm{c}}=4 \pi / 3 a^{2} b N$, where $N$ is the number of cracks per unit volume, behaves as $\mathrm{O}(\omega)$, so that the total contribution of the isotropically distributed opened cracks to the average strain is finite and given as a function of the crack density parameter $d=N a^{3}$ of [17] by the concentration tensor [21]:

$$
\lim _{\omega \rightarrow 0} f_{c}{\overline{\mathbb{L}_{c}^{0}}}^{c}=d \mathbb{Q}^{0}
$$


where $\boldsymbol{Q}^{0}$ is an isotropic fourth order whose components in the (J, K) basis are [22]:

$$
Q_{\mathrm{sph}}^{0}=\frac{4 k_{0}\left(3 k_{0}+4 \mu_{0}\right)}{3 \mu_{0}\left(3 k_{0}+\mu_{0}\right)} \quad ; \quad Q_{\mathrm{dev}}^{0}=Q_{\mathrm{sph}}^{0} \frac{4 \mu_{0}\left(9 k_{0}+4 \mu_{0}\right)}{15 k_{0}\left(3 k_{0}+2 \mu_{0}\right)}
$$

In turn, the contribution of isotropically distributed opened cracks to diffusion is accounted for by the average concentration gradient in cracks via:

$$
{\overline{L_{c}^{0}}}^{c}=\frac{D_{0} / 3}{D_{0}-S_{n}\left(D_{c}-D_{0}\right)}+\frac{2 D_{0} / 3}{D_{0}-S_{t}\left(D_{c}-D_{0}\right)} \quad \text { with } \quad 2 S_{t}=1-S_{n}=\frac{\pi}{2} \omega+o(\omega)
$$

To build estimates of the homogenized properties, local fields in each phase $i$ are estimated by (6a) for some appropriate choices of the reference medium $\boldsymbol{C}_{0}, \boldsymbol{D}_{0}$. The remote strain $\boldsymbol{E}_{\infty}$ and concentration gradient $\boldsymbol{\nabla} \boldsymbol{C}_{\infty}$ are determined be applying the averaging rule (1a). Eventually, the definition (5) yields:

$$
\mathbb{C}^{\text {hom }}=\overline{\mathbb{C}: \mathbb{L}^{0}}:{\overline{\mathbb{L}^{0}}}^{-1}=\left(\sum_{i \in\{p, s, c\}} f_{i} \mathbb{C}_{i}: \mathbb{L}_{i}^{0}\right):\left(\sum_{i \in\{p, s, c\}} f_{i} \mathbb{L}_{i}^{0}\right)^{-1} \quad ; \quad \boldsymbol{D}^{0}=\overline{\boldsymbol{D} \cdot \boldsymbol{L}^{0}} \cdot{\overline{\boldsymbol{L}^{0}}}^{-1}
$$

The Mori-Tanaka estimate assumes that one phase plays the specific role of a matrix, in which separate inclusions of other phases are embedded. For mortar, cement paste would be such matrix while micro-cracks and sand aggregates could be considered as various families of inclusions, so that one selects the reference medium to have the characteristics $\boldsymbol{C}_{0}=\boldsymbol{C}_{\mathrm{p}}$ and $\boldsymbol{D}_{0}=\boldsymbol{D}_{\mathrm{p}}$ in eq. (11), where by extension $\boldsymbol{L}_{\mathrm{p}}{ }^{\mathrm{p}}=\mathbf{I}$ and $\boldsymbol{L}_{\mathrm{p}}{ }^{\mathrm{p}}=\mathbf{1}$. In the Mori-Tanaka scheme, the inclusion phases are always assumed disconnected. In particular, cracks do not interconnect so that the homogenized stiffness is non zero even for a high density of cracks while the increase of diffusion coefficient is moderate as cracks develop.

Alternatively, the self-consistent estimate does not promote any phase to the role of a matrix. Each phase is treated by ellipsoidal inclusions immersed in the sought-for homogenized material, i.e. $\boldsymbol{C}_{0}=\boldsymbol{C}^{\text {hom }}$ and $\boldsymbol{D}_{0}=\boldsymbol{D}^{\text {hom }}$ so that the homogenized properties (11) are solutions to implicit equations. In that case, a spherical shape is attributed to cement paste. In the self-consistent scheme, any phase may create a connected domain, provided its volume fraction is above a percolation threshold which depends on all phases shapes (see e.g. [30]). In particular, it is possible to account for sharp stiffness loss or diffusion coefficient increase as cracks develop.

The generalization to poro-elasticity of the above estimates follows a similar treatment involving prestresses in the Eshelby problems, see [31-33]. Following [14, 31, 32], the Mori-Tanaka estimate of the poro-elastic properties of the mortar are:

$$
b_{m t}^{\mathrm{hom}}=f_{p} \operatorname{sph}\left(\overline{\mathbb{L}}^{-1}\right) \quad ; \quad 1 / N_{m t}^{\mathrm{hom}}=3 b_{m t}^{\mathrm{hom}} \operatorname{sph}\left(\overline{\mathbb{L}^{p}: \mathbb{P}^{p}}\right)
$$

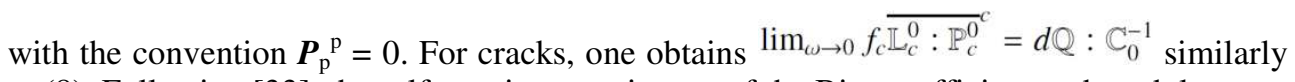
to (8). Following [33], the self-consistent estimates of the Biot coefficient and modulus are:

$$
\begin{aligned}
& b_{s c}^{\text {hom }}=\operatorname{sph}\left(f_{p} \mathbb{L}_{p}^{\text {hom }}:{\overline{\mathbb{L}^{\text {hom }}}}^{-1}\right) \\
& 1 / N_{s c}^{\text {hom }}=-3 \operatorname{sph}\left(f_{p} \mathbb{L}_{p}^{\text {hom }}:\left[{\overline{\mathbb{L}^{\text {hom }}}}^{-1}:\left(f_{p} \mathbb{L}_{p}^{\text {hom }}: \mathbb{P}_{p}^{\text {hom }}-\overline{\mathbb{L}^{\text {hom }}: \mathbb{P}^{\text {hom }}}: \mathbb{B}_{p}^{\text {hom }}\right)-\mathbb{P}_{p}^{\text {hom }}:\left(\mathbb{I}-\mathbb{B}_{p}^{\text {hom }}\right)\right]\right)
\end{aligned}
$$




\subsection{Application to mortar with normalized sand and CEM-I}

The evolution of the mortar properties w.r.t. the crack density parameter $d$ are estimated by the Mori-Tanaka and self-consistent schemes for a mortar comprising a CEM-I cement paste and normalized silica sand. The paste is assumed to be fully hydrated with a water to cement ratio equal to 0.5. Its stiffness parameters are taken from [34] and (chloride) diffusion coefficient from [35]. Silica sand is assumed non diffusive, while the diffusion coefficient of micro-cracks is assumed equal to the one of chloride in bulk water $D_{\text {bulk }}=$ $2.1 \times 10^{-9} \mathrm{~m} / \mathrm{s}^{2}$. Model input parameters used in figure 2 are summarized in table 1 . Initial sand and cement paste volume fractions are $f_{\mathrm{s}}=58 \%$ and $f_{\mathrm{p}}=42 \%$ [14]. Diffusion coefficients in figure $2 \mathrm{~b}$ are homogenized assuming a crack aspect ratio $\omega=1 \times 10^{-2}$ constant w.r.t. $d$.

Table 1. Model parameters.

\begin{tabular}{|c|c|c|c|}
\hline Phase & $\boldsymbol{k}_{\mathbf{i}}[\mathbf{G P a}]$ & $\boldsymbol{\mu}_{\mathbf{i}}[\mathbf{G P a}]$ & $\boldsymbol{D}_{\mathbf{i}} / \boldsymbol{D}_{\text {bulk }}$ \\
\hline Cement paste & 16 & 6 & $2.1 \times 10^{-3}$ \\
\hline Silica sand & 35 & 30 & 0 \\
\hline Microcracks & 0 & 0 & 1 \\
\hline
\end{tabular}

When the crack density parameter $d=0$, the self-consistent scheme $\left(E_{\mathrm{sc}}(0)=38.7 \mathrm{GPa}\right.$, $\left.D_{\text {sc }}(0)=2.7 \times 10^{-4} D_{\text {bulk }}\right)$ overestimates the Young's modulus $E$ and underestimates the diffusion coefficient of the undamaged mortar as it simulates a percolation of the sand grains $\left(E_{\text {exp }}(0)=34.5-36 \mathrm{GPa}[36-38], D_{\text {exp }}(0)=4.2 \times 10^{-3} D_{\text {bulk }}\right.$ [39] [with $\left.\left.f_{\mathrm{s}}=0.7\right]\right)$, whereas the Mori-Tanaka scheme accounts well for the matrix role played by cement paste $\left(E_{\mathrm{mt}}(0)=\right.$ $\left.34.8 \mathrm{GPa}, D_{\mathrm{mt}}(0)=6.8 \times 10^{-4} D_{\text {bulk }}\right)$. The underestimation of both schemes regarding the diffusion coefficient of the undamaged mortar is likely linked to the neglected ITZ between sand and paste.

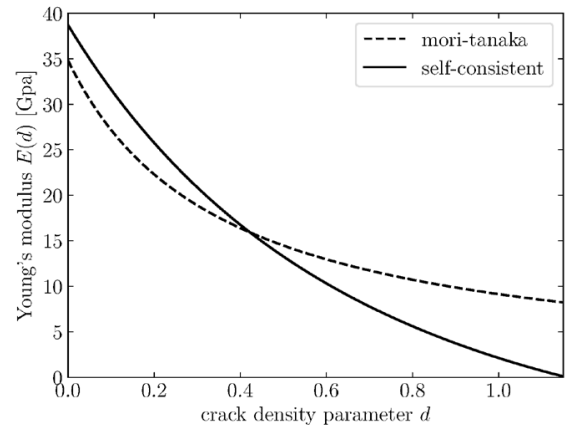

(a) Young's modulus

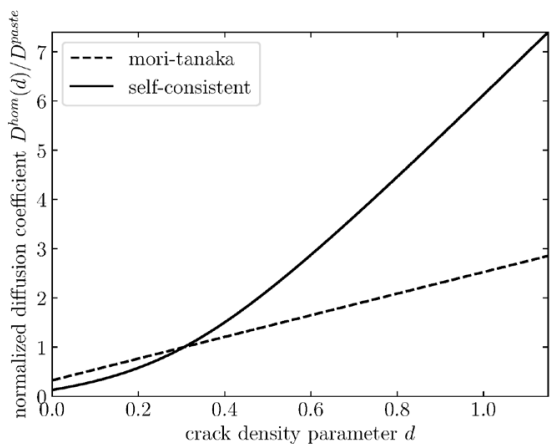

(b) diffusion coefficient

Fig. 2. Estimated evolution of Young's modulus and diffusion coefficient of mortar with crack density parameter $d$

Figure $2 \mathrm{~b}$ illustrates that the self-consistent scheme is more sensitive to the crack density parameter than the Mori-Tanaka one. Its estimates on the elastic moduli of micro-cracked media have been shown accurate at high crack densities [40]. As regards transport properties, the self-consistent scheme is also able to account for rapid changes in diffusion coefficient observed for micro-cracked media [23]. The estimates provided by both schemes on the increase of diffusion coefficient with mechanical damage are compared to experimental results by [6] on concrete sample damaged by uniaxial compression tests in figure 3. The apparent damage $1-E(d) / E(0)$ observed in experiments is computed from the ratio of damaged to undamaged Young's moduli. While tracking effects of relative 
diffusion coefficient rise w.r.t. damage propagation by microcracking, the self-consistent scheme thus appears more reliable than the Mori-Tanaka scheme, because it may account for microcracks percolation. The latter comparison is however to be considered with care as in the experiments of [6] damage induced by uniaxial compression likely induces anisotropically distributed cracks orientations, with crack aspect ratio depending on both load level and crack orientation w.r.t. load direction.

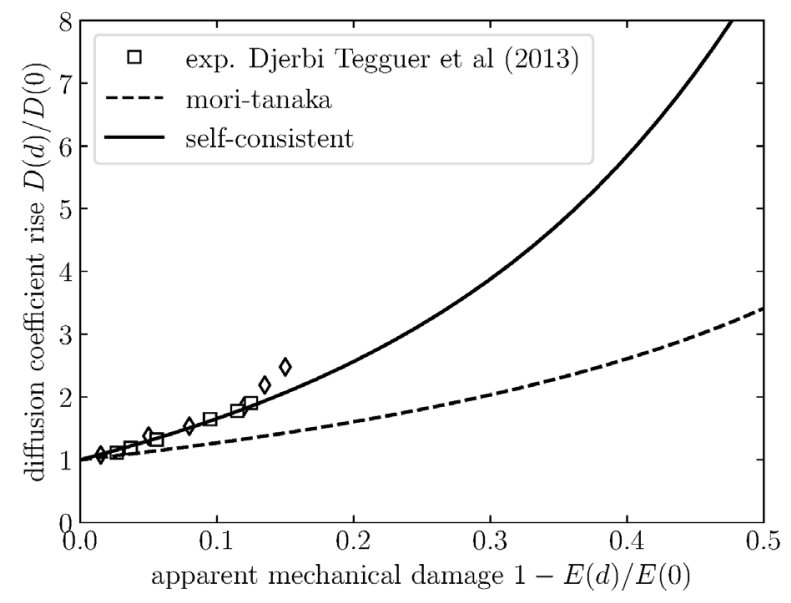

Fig. 3. Estimated diffusion coefficient increase with observable apparent damage.

\section{Damage due to microcracking development}

\subsection{Damage description and driving force}

Sect. 2 illustrates that the crack density parameter $d$ at the microscopic scale is a choice candidate for the description of the damage level of mortar. Damage evolution is accounted for from an energetic argument following [11, 19, 21-24, 41]. At the macroscopic scale, the potential energy per unit volume is [42]:

$$
\psi^{*}(\boldsymbol{E}, p, d)=\frac{1}{2} \boldsymbol{E}: \mathbb{C}^{\mathrm{hom}}(d): \boldsymbol{E}-\frac{p^{2}}{2 N^{\mathrm{hom}}(d)}-p b^{\mathrm{hom}}(d) \mathbf{1}: \boldsymbol{E}
$$

In a reversible evolution of the state variables $(\boldsymbol{E}, p)$, the potential energy appears as a potential for the macroscopic poroelastic constitutive law (4). In an irreversible evolution, the energy dissipation is $\quad-\dot{d} \partial \Psi^{*} / \partial d \geq 0$. The damage driving force is then:

$$
\mathcal{F}_{d}=-\frac{\partial \Psi^{*}}{\partial d}=\frac{1}{2} \boldsymbol{E}: \frac{\partial \mathbb{C}^{\text {hom }}}{\partial d}: \boldsymbol{E}-\frac{p^{2}}{2} \frac{\partial}{\partial d}\left(\frac{1}{N^{\text {hom }}}\right)-p \frac{\partial b^{\text {hom }}}{\partial d} \mathbf{1}: \boldsymbol{E}
$$

The partial derivatives w.r.t. $d$ in (14) are readily obtained from the homogenization procedure described in Sect. 2. 


\subsection{Damage propagation criterion}

By analogy with the energy release rate in linear elastic fracture mechanics (LEFM), damage propagation can be described by a criterion [22]:

$$
\mathcal{F}_{d}-\mathcal{F}_{c} \leq 0 \quad ; \quad \dot{d} \geq 0 \quad ; \quad\left(\mathcal{F}_{d}-\mathcal{F}_{c}\right) \dot{d}=0
$$

where $F_{\mathrm{c}}$ is an analogue to the fracture energy in LEFM. However $F_{\mathrm{c}}$ is a priori not an intrinsic material property and could depend on the scale of observation and loading history via the history of development of the cracking pattern at the microscopic scale.

From a purely macroscopic point of view, the simplest choice is to assume $F_{\mathrm{c}}$ independent on $d[21-23,41]$ or linear w.r.t. $d[20,24]$ :

$$
\mathcal{F}_{c}(d)=\mathcal{F}_{c, 0}(1+B d)
$$

where $F_{\mathrm{c}, 0}$ and $B$ are constants. Alternatively, one may assume that the number of microcracks per unit volume $N$ is constant and that all cracks have the same increasing length $a$ as damage increases. Analogy with LEFM, where $G_{c}$ the surface fracture energy at the crack scale, leads to [19]:

$$
\mathcal{F}_{c}(d)=\frac{2 \pi}{3 a} G_{c}=\frac{2 \pi \mathcal{N}^{1 / 3}}{3 d^{1 / 3}} G_{c}
$$

\subsection{Application to damage induced by DEF}

A series of experiments [36-38] have studied the macroscopic expansion along with measurement of Young's modulus loss over months of samples subjected to DEF. In these experiments, the macroscopic stress $\Sigma=0$ so that the isotropic macroscopic expansion $\boldsymbol{E}=$ $E_{\mathrm{DEF}} \mathbf{1}$ is linked to the DEF pressure in cement paste $p=3 k_{\mathrm{p}} \varepsilon_{\mathrm{f}}$ by (4) as $E_{\mathrm{DEF}}=$ $\left(b^{\text {hom }} / 3 k^{\text {hom }}\right) p$. The potential energy is then only function of the two state variables $(p, d)$ and the damage driving force simplifies to

$$
\mathcal{F}_{d}=p^{2} \mathcal{G}(d) \quad \text { with } \quad \mathcal{G}(d)=\frac{1}{2} \frac{\partial}{\partial d}\left(\frac{1}{N^{\text {hom }}}+\frac{\left(b^{\text {hom }}\right)^{2}}{k^{\text {hom }}}\right) .
$$

The mortar follows a reversible poroelastic evolution until the DEF pressure reaches a first value such that $F_{\mathrm{d}}=F_{\mathrm{c}}$. The evolution is then irreversible and the damage $d$ evolution law yields $p=\left(F_{\mathrm{c}}(d) / G(d)\right)^{1 / 2}$.

The various of expressions of $F_{\mathrm{c}}(d)$ in (16) and (17) are now evaluated in the case of damage induced by DEF. The assumption of a constant number of growing cracks leading to (17) with a decreasing $F_{\mathrm{c}}(d)$ results in an unstable evolution of the damage, which contradicts experiments. Two other possibilities summed up by (16), one with $F_{\mathrm{c}}$ constant $(B=0)$ and the other one with $F_{\mathrm{c}}$ increasing $(B>0)$ are illustrated in figure 4 . For illustration purposes, model parameters in (16) are adjusted to $F_{\mathrm{c}, 0}=5 \mathrm{MPa}, B=0$ in the first case and to $F_{\mathrm{c}, 0}=0.1 \mathrm{MPa}, B=400$ in the second case. The assumption in [21-23, 41] of a constant value of $F_{\mathrm{c}}$ leads to a reversible evolution with no damage up to unrealistic values of the macroscopic expansion, followed by rapid damage propagation. This behaviour is exacerbated with the self-consistent estimate. In turn, the assumption in $[20,24]$ of an increasing $F_{\mathrm{c}}(d)$ allows to simulate an early initiation of damage followed by a slower damage propagation. Simulated trends are in better agreement with experimental results. 


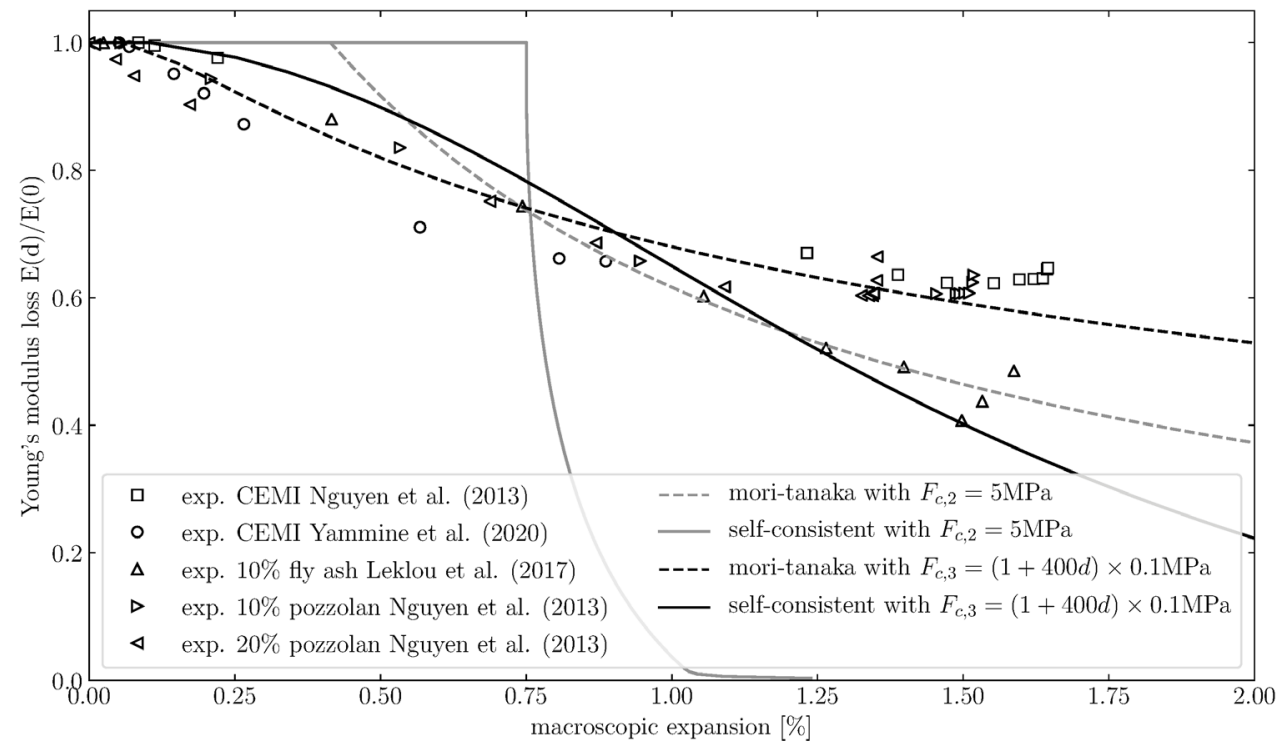

Fig. 4. Modeled Young's modulus loss as a function of macroscopic expansion as compared to experimental data from [36-38]

\section{Conclusion}

A multi-scale model has been developed to account for damage induced by DEF under the assumption of a uniform free expansion of cement paste. The model accounts explicitly for the elastic moduli decrease and diffusion coefficient increase as expansion develops via micro-cracking development at the aggregate scale. Among the two homogenization schemes investigated, the Mori-Tanaka one provides a better agreement with experimental results on an undamaged mortar, but the self-consistent scheme appears more suitable to model the rapid coefficient of diffusion increase and Young's modulus loss as damage develops.

The effect of the mathematical dependence of the damage propagation criterion on the damage variable has been investigated. Comparison to experimental results at the macroscopic scale indicates that constant or decreasing values of the critical energy release rate leads to unrealistically late or unstable damage propagation. In turn, the assumption of an increasing critical energy release rate yields a much more satisfactory evolution of damage with expansion, for both homogenization schemes.

An acknowledged limitation of the method lies in the absence of a clear link between the critical energy release rate and the microstructure parameters and configuration. Its likely dependence on loading type and history renders it difficult to measure from direct observations, which impairs the predictability of the model. Further, the duration of DEF over several months would require the consideration of creep.

The authors acknowledge the Algerian-French cooperation project Tassili 19MDU216 and the financial support of the Polish National Agency for Academic Exchange under the International Academic Partnership Programme: PPI/APM/2018/1/00027 E-mobility and sustainable materials and technologies EMMAT. 


\section{References}

1. S. Diamond, Cem. Conc. Comp. 18, 205 (1996)

2. K.L. Scrivener, D. Damidot, C. Famy, Cem. Conc. Aggr. 21, 93 (1999)

3. H. Taylor, Ceramic Transactions, 4061 (1994)

4. G.W. Scherer, International RILEM Workshop on Internal Sulfate Attack and Delayed Ettringite Formation 139-154 (2004)

5. X. Brunetaud, Ph.D. thesis, Ecole centrale de Paris (2005)

6. A. Djerbi Tegguer, S. Bonnet, A. Khelidj, V. Baroghel-Bouny, Cem. Concr. Res. 52 (2013)

7. B. Bary, Int. J. Numer. Anal. Meth. Geomech. 32, 1791 (2008)

8. M. Basista, W. Weglewski, Int. J. Dam. Mech. 18, 155 (2009)

9. A. Sellier, S. Multon, Cem. Concr. Res. 108, 72 (2018)

10. L. Charpin, A. Ehrlacher, Cem. Concr. Res. 63, 143 (2014)

11. R. Esposito, M. Hendriks, Cem. Concr. Comp. 70, 139 (2016)

12. T. Iskhakov, J.J. Timothy, G. Meschke, Cem. Concr. Res. 115, 507 (2019)

13. S. Multon, A. Sellier, Int. J. Sol. Struct. 160, 293 (2019)

14. A. Yammine, F. Bignonnet, N. Leklou, M. Choinska, T. Stryszewska, FraMCoS-X: 10th Int. Conf. on Fracture Mechanics of Concrete and Concrete Structures (2019)

15. A.I. Cuba Ramos, C. Roux-Langlois, C.F. Dunant, M. Corrado, J.F. Molinari, Cem. Concr. Res. 109, 90 (2018)

16. Y. Wang, P. Jiradilok, K. Nagai, S. Asamoto, Eng. Fract. Mech. 232, 107055 (2020)

17. B. Budiansky, R.J. O’Connell, Int. J. Sol. Struct. 12, 2 (1975)

18. L. Dormieux, E. Lemarchand, J. Eng. Mech. 127, 1267 (2001)

19. L. Dormieux, D. Kondo, F.J. Ulm, C. R. Mecanique 334, 440 (2006)

20. Q. Zhu, D. Kondo, J. Shao, Int. J. Sol. Struct. 45, 1385 (2008)

21. L. Dormieux, D. Kondo, Int. J. Mech. Sci. 90, 25 (2015)

22. L. Dormieux, D. Kondo, Micromechanics of Fracture and Damage, John Wiley \& Sons (2016)

23. L. Dormieux, D. Kondo, C. R. Mecanique 332, 135 (2004)

24. J.F. Shao, H. Zhou, K.T. Chau, Int. J. Num. Anal. Meth. Geomech. 29, 1231 (2005)

25. T. Jiang, J. Shao, W. Xu, C. Zhou, Int. J. Rock Mech. Mining Sci. 47, 703 (2010)

26. G. Caratini, Ph.D. thesis, Ecole des Ponts ParisTech, Université Paris Est (2012)

27. Y. Chen, S. Hu, C. Zhou, L. Jing, Rock Mech Rock Eng 47, 1775 (2014)

28. A. Zaoui, J. Eng. Mech. 128, 808 (2002)

29. J.D. Eshelby, Proc. Roy. Soc. London. A: Math. Phys. Sci. 241, 376 (1957)

30. M. Achour, F. Bignonnet, J.F. Barthélémy, E. Rozière, O. Amiri, Constr. Build. Mat. 234, 117124 (2020)

31. Y. Benveniste, G. Dvorak, T. Chen, J. Mech. Phys. Solids 39, 927 (1991)

32. B. Pichler, L. Dormieux, C. R. Mecanique 336, 636 (2008)

33. B. Pichler, C. Hellmich, J. Eng. Mech. 136, 1043 (2010)

34. R. Helmuth, D. Turk, Portland Cement Association. Research and Development Laboratories (1966) 
35. C.L. Page, N.R. Short, A.E. Tarras, Cem. Concr. Res. 11, 395 (1981)

36. N. Leklou, V.H. Nguyen, P. Mounanga, KSCE J. Civil Eng. 21, 1359 (2017)

37. V.H. Nguyen, N. Leklou, J.E. Aubert, P. Mounanga., Constr. Build. Mat. 48, 479 (2013)

38. A. Yammine, N. Leklou, M. Choinska, F. Bignonnet, J.M. Mechling, Constr. Buil. Mat. 252, 119059 (2020)

39. C.C. Yang, Cem. Concr. Res. 36, 1304 (2006)

40. F. Willot, H. Trumel, D. Jeulin, Philosophical Magazine 99, 606 (2019)

41. L. Dormieux, D. Kondo, Poroelasticity and damage theory for saturated cracked media, Springer Vienna 153-186 (2005)

42. L. Dormieux, A. Molinari, D. Kondo, J. Mech. Phys. Sol. 50, 2203 (2002) 